\title{
Bacteroides ureolyticus
}

National Cancer Institute

\section{Source}

National Cancer Institute. Bacteroides ureolyticus. NCI Thesaurus. Code C86181.

A species of anaerobic, Gram-negative, rod shaped bacteria assigned to the phylum

Bacteroidetes. This species is nonmotile, non-spore forming, urease positive, catalase negative, oxidase positive, does not produce indole, hydrolyzes gelatin and casein but not starch, reduces nitrate, produces hydrogen sulfide, and does not ferment carbohydrates.

B. ureolyticus is pathogenic in humans. 